\title{
Gestão e liderança na percepção de enfermeiros: um caminhar à luz da burocracia profissional
}

\author{
Management and leadership in the nurses' perception: A walk in the light of professional burocracy \\ Gestión y liderazgo en la percepción de los enfermeros: un paseo a la luz de la burocracia profesional
}

Gilberto Tadeu Reis da Silva ${ }^{1}$ (B)

Patrícia Alves Galhardo Varanda ${ }^{1}$ (1) Nívia Vanessa Carneiro dos $\operatorname{Santos}^{1}$ (]) Naomy Safira Batista da Silva² (B) Rilary Silva Salles ${ }^{2}$ (1) Simone Coelho Amestoy ${ }^{1,3,4}$ (D) Giselle Alves da Silva Teixeira ${ }^{2}$ (1) Paulo Joaquim Pina Queirós ${ }^{5}$ (B)

1. Universidade Federal da Bahia, Programa de Pós-Graduação em Enfermagem e Saúde. Salvador, BA, Brasil.

2. Universidade Federal da Bahia, Escola de Enfermagem. Salvador, BA, Brasil.

3. Universidade Federal de Pelotas, Faculdade de Enfermagem. Pelotas, RS, Brasil.

4. Universidade Federal do Vale do São Francisco. Petrolina, PE, Brasil.

5. Escola Superior de Enfermagem de Coimbra. Coimbra, Portugal.

\section{Resumo}

Objetivo: analisar os estilos e fatores intervenientes na gestão e liderança de enfermeiros em três países, Brasil, Portugal e Espanha, à luz da Burocracia Profissional. Método: estudo exploratório e descritivo de abordagem qualitativa realizado em três hospitais universitários localizados em diferentes países: Brasil, Espanha e Portugal. Participaram da pesquisa 30 enfermeiros. Os dados foram coletados por meio de um roteiro de coleta de dados com questões sociodemográficas e entrevistas analisadas com auxílio do software WebQda. Resultados: diferentes percepções sobre os elementos integradores da participação foram reveladas nos três países, destacando-se a comunicação em diversas óticas. Verificou-se algumas convergências em relação ao trabalho em equipe, sendo a confiança o elemento que impulsiona e motiva a equipe. Sinaliza-se para uma relação participativa no desenvolvimento do trabalho. Conclusão e implicações para a prática: foi possível identificar, nos três países, a importância da comunicação no processo de gestão, bem como dos estilos de gestão e de liderança, como elementos que oportunizam a atuação da equipe. Também foi evidenciada a presença de fatores intervenientes de relevância, tais como escuta, clima organizacional relação interpessoal, transparência no trabalho e delegação de funções, os quais envolvem a burocracia profissional em que o conhecimento do enfermeiro possibilita o exercício de suas habilidades de forma horizontalizada e participativa.

Palavras-chave: Enfermagem; Liderança; Capacidade de Liderança e Governança; Gestão em Saúde; Equipe de Enfermagem.

\section{Abstract}

Objective: to analyze the styles and factors involved in nurses' management and leadership in three countries, Brazil, Portugal and Spain, in the light of Professional Bureaucracy. Method: a descriptive and exploratory study with a qualitative approach carried out in three university hospitals located in different countries, namely: Brazil, Spain and Portugal. Thirty nurses participated in the research. Data was collected through a collection script with sociodemographic questions and interviews analyzed using the WebQda software. Results: different perceptions about the integrating elements of participation in the three countries were revealed, highlighting communication from different perspectives. Some convergences in relation to teamwork were verified, where trust was the element that drives and motivates the team. It is a participatory relationship in the development of work. Conclusion and implications for the practice: in the three countries, it was possible to identify the importance of communication in the management process, as well as the management and leadership styles, as elements that favor performance of the team. Presence of relevant intervening factors was also evidenced, such as choice, organizational climate, interpersonal relationships, transparency in work and delegation of functions, which involves professional bureaucracy in which the nurses' knowledge allows them to exercise their skills in a horizontal and participatory manner.

Keywords: Nursing; Leadership; Leadership and Governance Capacity; Health Management; Nursing Team.

\section{Resumen}

Objetivo: analizar los estilos y factores implicados en la gestión y el liderazgo de enfermeros en tres países, Brasil, Portugal y España, a la luz de la Burocracia Profesional. Método: estudio descriptivo exploratorio con abordaje cualitativo realizado en tres hospitales universitarios ubicados en diferentes países: Brasil, España y Portugal. Treinta enfermeros participaron en la investigación. Los datos fueron recolectados a través de un script de recopilación de datos con preguntas sociodemográficas y entrevistas analizadas con la utilización del software WebQda. Resultados: se revelaron diferentes percepciones sobre los elementos integradores de la participación en los tres países, destacando la comunicación desde diferentes perspectivas. Se constataron algunas convergencias en relación al trabajo en equipo, siendo la confianza el elemento que impulsa y motiva al equipo. Se advierte una relación participativa en el desarrollo del trabajo. Conclusión e implicaciones para la práctica: se pudo advertir, en los tres países, la importancia de la comunicación en el proceso de gestión, así como los estilos de gestión y liderazgo, como elementos que permiten mejorar el desempeño del equipo. También se evidenciaron factores intervinientes relevantes, como la escucha, el clima organizacional, la relación interpersonal, la transparencia en el trabajo y la delegación de funciones, que conforman la burocracia profesional en la cual el conocimiento de los enfermos les permite el despliegue de sus competencias de manera horizontal y participativa.

Palabras clave: Enfermería; Liderazgo; Capacidad de Liderazgo y Gobernanza; Gestión en Salud; Equipo de Enfermería. 


\section{INTRODUÇÃO}

O processo de trabalho do enfermeiro requer o exercício da liderança desde a gestão da equipe de enfermagem até outros níveis organizacionais, como a gerência do serviço de saúde. Desse modo, considerando as teorias de liderança, desde as mais clássicas até as mais contemporâneas, define-se liderança como uma competência necessária ao enfermeiro, tornando-o capaz de influenciar sua equipe com vistas a assegurar atendimento focado nas reais necessidades de saúde dos usuários e familiares ${ }^{1}$.

Mesmo com diversas perspectivas teóricas, ainda não há uma definição consensual acerca do conceito de liderança, uma vez que as múltiplas teorias atribuem diferentes significados a essa competência. Na Enfermagem, a liderança está relacionada com as ações do enfermeiro como líder do processo de cuidar e exemplo a ser seguido por sua equipe de enfermagem. Alicerçada também na relação de confiança e horizontalidade construída no respeito mútuo, nas atitudes justas e flexíveis de suas ações, em que oportuniza a participação dos colaboradores nas tomadas de decisões ${ }^{2,3}$.

$\mathrm{Na}$ dimensão gerencial, o processo de trabalho do enfermeiro, visa assegurar a qualidade da assistência e um o bom funcionamento dos serviços de saúde, que requerem do profissional não só o dinamismo como capacidade de análise crítica, diálogo e vínculo com a equipe de saúde. A atuação do enfermeiro gestor, pelo Conselho Federal de Enfermagem, institui atributos de chefia, coordenação, planejamento, organização e avaliação das atividades de enfermagem, como importantes ferramentas para o exercício gerencial do enfermeiro ${ }^{4}$.

Esse processo de trabalho se diversifica em distintas realidades, entretanto, o componente gerencial surge em comum entre países como Brasil, Estados Unidos, Canadá, Chile, Austrália, Portugal, Reino Unido, Japão, África do Sul, China e Tailândia. Observa-se como similaridade uma ocultação da dimensão gerencial por razões ideológicas em que as atribuições do enfermeiro estão restritas a atividades assistenciais e pela divisão técnica do trabalho na enfermagem ${ }^{5}$.

Ressalta-se que em sistemas sociais complexos, como os existentes na Europa, a liderança apresenta-se inerente às atividades gerenciais, e têm sido vista como potencializadora dos resultados dos cuidados de enfermagem ${ }^{6}$.

O processo de gerenciar equipes, comum à realidade do enfermeiro, requer devida atenção, pois cada cenário em que este profissional está inserido possui uma característica específica, de modo que, por vezes, é preciso trabalhar a própria ideia de equipe, reforçando o trabalho interdependente ${ }^{7}$.

Cabe mencionar que estudo realizado em Portugal sinalizou que as lideranças nos diferentes serviços se encontram frente ao desafio de gerir e coordenar os saberes e fomentar as competências e o desempenho dos colaboradores. Assim sendo, o serviço de saúde cria sistemas de qualidade e em acordo com o pressuposto pela Ordem dos Enfermeiros Portugueses, onde as ações dos enfermeiros líderes são respeitadas e ganham destaque ${ }^{8}$.

$\mathrm{Na}$ Espanha, uma pesquisa observou que o excesso de atividades gerenciais e administrativas impostas ao trabalho do enfermeiro o afasta da relação com paciente. Isso denota a preocupação desses profissionais com o tempo dedicado no cuidado do paciente, e evidencia que o processo de gerenciamento do trabalho em saúde, do modo como vem sendo planejado e executado, acaba dificultando o cuidar. Ressalta também alguns aspectos essenciais para adquirir eficácia na assistência como as relações humanas saudáveis entre os profissionais, pacientes e familiares, estar satisfeito com o trabalho, ser reconhecido e aceito na equipe de trabalho, assim como criar laços de confiança com a mesma9.

Convém ressaltar que, no Brasil, há o destaque das competências e habilidades do enfermeiro durante o processo de formação, estando presentes a comunicação, a liderança, a administração, o gerenciamento e a tomada de decisões, que possibilitam o desenvolvimento adequado desde a graduação, expressar em recomendações do Conselho Nacional de Saúde frente as diretrizes curriculares nacionais propostas. $O$ mercado de trabalho e as inovações técnico-científicas também corroboram a necessidade do aperfeiçoamento dos enfermeiros, uma vez que visam altas performances e desempenhos fundamentados na inteligência e personalidade das pessoas ${ }^{10}$.

Uma das teorias que contribuem para essa perspectiva de aperfeiçoamento constante, pautado na realidade, é o modelo de Burocracia Profissional proposto por Henry Mintzberg. Tal modelo defende que as habilidades e o conhecimento das profissões são programados formalmente de acordo com o que é esperado do profissional, seguido por um longo tempo de prática nos serviços, levando-o a desenvolver novos conhecimentos e habilidades ${ }^{11}$. Assim, a gestão com base nesta perspectiva pode ser compreendida por capacidades específicas e diferenciadas adquiridas mediante a vivência profissional e os conhecimentos alcançados, já a liderança é um processo de evolução constante, que parte do conhecimento científico e da imersão no cotidiano de trabalho.

Em síntese, parte-se do princípio de que a liderança é uma competência fundamental para o enfermeiro em sua prática profissional reconhecendo os diversos estilos de liderança existentes em diferentes países. Além disso, a gestão é essencial para a eficácia na assistência de enfermagem ambas são imprescindíveis para a harmonia no trabalho em equipe, o que exige do profissional sua continua atualização.

Portanto, a escolha dos três países permitiu a apreensão de distintas realidades, pensando nas convergências e divergências, que se expressam por meio dos modelos e práxis de cada país. Considerou-se que a qualidade no cuidado ao usuário por meio da gestão e liderança do enfermeiro consiste em uma realidade compartilhada por eles. Frente ao exposto, o presente trabalho tem por objetivo analisar os estilos e fatores intervenientes na gestão e liderança de enfermeiros em três países, Brasil, Portugal e Espanha, à luz da Burocracia Profissional.

\section{MÉTODO}

Estudo exploratório e descritivo de abordagem qualitativa, associado à investigação intitulada "Modelos de Gestão em 
Enfermagem: Memórias de Enfermeiras". A abordagem qualitativa estabelece ligações entre o fenômeno a ser estudado e conceitos, representações, crenças e comportamentos ${ }^{12}$.

Os cenários do estudo foram três hospitais universitários: no Brasil, Espanha e Portugal. A opção por esses serviços ancora-se em que ambos os países desde a década de 90 têm adotado políticas públicas de proteção à saúde como direito de cidadania por meio de sistemas públicos e universais de saúde. Destacamos, ainda, um investimento significativo na formação em gestão em saúde para qualificar e capacitar os gestores públicos. Ademais, em consonância com os estudos destes autores ${ }^{13,14}$, os serviços nacionais de saúde dos países indicados têm implementado programas de melhoria da eficiência e eficácia na gestão dos hospitais universitários.

Utilizou-se a técnica de Snowball Sampling, em que uma amostragem não probabilística emprega cadeias de referências. Tal método é empregado quando se estudam características raras ou quando se busca acesso a grupos sociais mais restritos ${ }^{15}$.

A seleção dos participantes do estudo ocorreu através de convites a profissionais que atendessem os critérios de elegibilidade. Ao total, foram convidados para a pesquisa 39 indivíduos, mas dos indicados na técnica utilizada somente 30 profissionais aceitaram participar e, dessa maneira, foram incluídos na investigação. Utilizou-se como critérios de elegibilidade enfermeiros que exerciam gestão assistencial, intermediária e de direção, em diferentes níveis, com atuação no serviço há mais de dois anos.

A coleta de dados ocorreu em três momentos interdependentes, sendo o primeiro Portugal, o segundo Espanha e o terceiro o Brasil, através de entrevistas norteadas por um roteiro de coleta de dados pré-estruturado, com a participação do investigador principal ou, quando necessário, de uma colaboradora.

As entrevistas foram realizadas no período de setembro de 2019 a fevereiro de 2020 , em local e espaço reservados previamente pelos entrevistados para esta finalidade. O roteiro foi dividido em duas partes, sendo a primeira parte formada por questões sociodemográficas como sexo, estado civil, raça/cor autodeclarada, escolaridade, tempo de formação, instituição de formação em nível superior, carreira de gestão em anos (Sindicato, Associação, Conselho, Partido Político), tempo total de carreira em gestão, religião, renda salarial e área de atuação profissional na enfermagem visando caracterizar os participantes do estudo. $\mathrm{Na}$ segunda fase, foram utilizadas as questões norteadoras específicas do objeto de estudo. A opção deste instrumento de coleta aconteceu por conta do direcionamento efetivo que ele fornece, além de proporcionar fluidez no diálogo e permitir ao entrevistado reflexão sobre os tópicos abordados ${ }^{15}$.

As entrevistas foram gravadas com auxílio de um modelo de aparelho celular que agrega, também, algumas funções dos computadores, cujos sistemas operacionais são adaptados para esse tipo de equipamento. Em seguida, as entrevistas foram transcritas, avaliadas em termos de coerência e organizadas no programa WebQda, a fim de identificar a frequência absoluta e relativa das categorias de análise do material.
Após a organização dos dados neste software, os textos foram organizados em núcleos de sentidos e agregados em categorias autoexcludentes em consonância com a proposta operativa de Minayo ${ }^{12}$, para organização e categorização na busca do material empírico.

No intuito de preservar o anonimato dos participantes, estes foram identificados pelo nome do país de origem (Brasil, Espanha, Portugal) acrescido de um número correspondente da maneira de exemplo como segue: REF $1 / B R$, referente ao Brasil, Portugal REF1/ PORT e Espanha REF¹/ESP.

No atinente aos preceitos éticos, o projeto matriz foi aprovado pelo Comitê em Ética em Pesquisa (CEP), sob CAAE 15084819.4.0000.5531 ainda no atinente a estes necessários se faz expressar que os hospitais universitários que participaram do estudo acolheram a aprovação no Brasil, tendo sido exigida a tradução do Termo de Consentimento Livre Esclarecido para outro idioma.

\section{RESULTADOS}

A amostragem foi constituída predominantemente por profissionais do gênero feminino (88,9\%), sendo $11,1 \%$ do gênero masculino, com idades entre 32 e 67 anos, 55,6\% de raça/cor autodeclarada parda, $66,7 \%$ casadas(os), $66,7 \%$ com ensino superior e pós-graduação, com tempo médio de formação entre 09 a 37 anos, 66,7\% formou-se em uma instituição pública de ensino. Nenhum dos participantes teve carreira na gestão em Sindicatos, Associação, Partido Político, foi observado que o tempo total de carreira em gestão nos serviços de saúde variou entre 06 meses a 27 anos. As áreas de atuação em enfermagem elencadas pelos enfermeiros foram assistência, ensino, pesquisa, gestão, assessoria, educação continuada, auditoria, empreendimento, consultoria, coaching, palestrante e coordenação de pós-graduação.

Os resultados foram categorizados conforme o conteúdo das falas dos participantes de cada país, como se trata de países diferentes, optou-se em manter o idioma original de Portugal e Brasil, já as entrevistas da Espanha, para facilitar a leitura, foram traduzidas e validadas pelos participantes. As categorias que se destacaram foram: A integração da gestão e liderança no processo de trabalho do enfermeiro; Trabalho em equipe como esteio no processo de gerir/liderar e diferentes modelos de gestão e liderança no cuidado.

\section{A integração da gestão e liderança no processo de trabalho do enfermeiro}

Na primeira categoria, destacam-se aspectos da liderança durante o processo de trabalho do enfermeiro, como influenciar, dialogar, ouvir, motivar e desenvolver a equipe. Também aponta atividades de gestão ao supervisionar, planejar e implementar ferramentas para a qualidade do cuidado. Desta forma, emerge uma integração entre a gestão e a liderança no cotidiano de enfermagem, que mesmo sendo distintas são indissociáveis. Os participantes mencionaram também uma comunicação adequada entre equipe, paciente e família como elemento 
facilitador de um cuidado participativo. Além disso, destacaram a importância da inserção da equipe nas decisões da gestão para manutenção do engajamento e motivação nos projetos, da educação permanente com temáticas emergentes na prática profissional e do envolvimento da gestão no cotidiano da equipe como aspectos que contribuem para um ambiente de trabalho resolutivo.

Proximidade, não há supervisão se não estar próximo[...] não preciso de estar a vigiar alguém para ver se estar a fazer mal, eu vou estar, eu estou com a pessoa, e aproveito esses momentos para dizer, influir, influenciar sobre o que se faz e que não se deve fazer, influenciar da forma como falamos da forma que se tem a falar, muitas vezes nós falamos e não ajudamos a pessoa [...] (REF3/PORT).

No Brasil, nota-se como a gestão e a liderança se complementam no cotidiano de enfermagem. As falas expressam um processo de gestão focado no planejamento e uma liderança voltada para o desenvolvimento, treinamento e motivação da equipe, a fim de proporcionar a participação coletiva da equipe em atividades que promovam a qualidade da assistência, tal como se observa nos fragmentos a seguir:

[...] tinha um Núcleo, na ocasião chamava-se Núcleo TQC de controle da qualidade total, e eu que elaborei todo o planejamento para implantar o processo de qualidade, treinei todos os profissionais em todas as ferramentas da qualidade, fazia toda a motivação e a mobilização das pessoas para, absorverem e implantarem, participarem do processo coletivo de construção de um novo modelo de gestão em um hospital universitário [...] (REF¹/BR).

[...]Os processos educativos, a gente também não tinha nada, da divisão para cá, mas a gente, quando a gente montou a unidade vimos a necessidade disso, de criar, a gente faz treinamentos, muitos treinamentos em lócus, a gente incentiva a participação de congressos, porque tem muita coisa que a gente faz na unidade e a gente precisa divulgar [...](REF6/BR).

$\mathrm{Na}$ Espanha, evidenciaram-se elementos da liderança associados às relações interpessoais, reforçando que o trabalho gerencial do enfermeiro ultrapassa a dimensão institucional. Destacando-se o diálogo e o saber ouvir, a preocupação do enfermeiro em orientar o paciente e seus familiares, iniciando o acolhimento com orientações desde o momento de recepcionálos e durante todo o processo de internação:

Se eu vou receber uma internação aguda naquele dia e me apresento para a família, eu me apresento para o paciente, explico o funcionamento da unidade, falo para o paciente o que a gente vai fazer, converso um pouco com a família, conto onde estarei mesmo que eles já saibam bem que não vão me encontrar no escritório que estarei mais na unidade, estou mais de estar lá [...] (REF/ESP).

[...]Basta sentar e ouvir os pacientes, ou sentar e ouvir e falar, parece que a gente começa esse tratamento, e ébom porque, a gente passa segurança, a gente passa ajuda, que às vezes o que ele quer é ajuda, não uma técnica que é feita e agora que não tem acompanhamento, o primário é um acompanhamento do paciente com o qual é gentil que gera um bem-estar alheio a uma patologia que ele tem, mas a patologia melhora quando alguém tem atendimento 24 horas[...] (REF¹/ESP).

\section{Trabalho em equipe como esteio no processo de gerir/liderar}

Na segunda categoria, emergiu das entrevistas a importância do saber ouvir e envolver a equipe, por meio da criação de laços de confiança e da corresponsabilização dos profissionais com o serviço a ser prestado, o que impulsiona e fortalece as relações interpessoais e motiva a equipe. Os depoimentos a seguir evidenciam essa postura:

[...] Foi um momento de eu acolher, ouvir [...] eu tinha que ouvir das pessoas o que estava acontecendo, o que elas estavam sentindo, para daí ver o que eu poderia trabalhar, o que eu poderia ajudar. Foi aquele momento de acolhimento e junto com eles eu fui elaborando aquilo que poderia melhorar dentro do contexto da divisão de enfermagem, foi que eu comecei também, até para conhecer a minha equipe, porque para trabalhar também precisa conhecer as pessoas [...] (REF²/BR).

[...] eu prefiro ouvir as pessoas, envolvê-las, responsabilizálas, que eu sei a partir daí tenha a audição, se for dizer alguma coisa diz, fora do grupo, eu quero um grupo a funcionar, não quero vários grupos com cada um ao seu lado, quero que o grupo vista a camisola, ponha a bandeira, empunha a bandeira e que, de fato, tenha um sentido de espirito de equipe e de trabalho sustentado com o horizonte, com o objetivo definido. [...] (REF/PORT).

$\mathrm{Na}$ Espanha, os participantes referiram compreender a importância da gestão e do trabalho conjunto, principalmente com a equipe multidisciplinar:

A verdade é que desde que estou na gestão nesse sentido, por um lado, eu acredito que os supervisores existem para trabalhar com a equipe multidisciplinar [...] (REF²/ESP).

Para trabalhar, a gente tem que fazer uma aproximação com as ações de cuidado, as auxiliares e as demais enfermeiras têm que cumprir isso[...], a gente compartilha coma auxiliar porque, talvez, ela também tenha informações que eu não conheço e ela pode me passar com todos nós, nós compartilhamos isso[...] (REF³/ESP). 
A cooperação entre os profissionais de saúde, de modo que a equipe consolide seus esforços e ofereça apoio mútuo, foi destacada em Portugal:

Então neste momento na equipe, a minha preocupação em termos formativos foi criar peritos quase em todas as áreas, [...] portanto, os grupos já estão a trabalhar, a produzir conhecimento e a trabalhar nesta área, e os colegas já sabem se tiverem dúvida naquela área vão consultar o tal, e, portanto, é um pouco assim que eu faço a organização da formação (REF/PORT).

Isto acontece frequentemente com coisas muito pequeninas, o que eu tento fazer, recorrer ao alguém que seja mais expert nessa área e tentar ajudar na altura, e funciona muito bem, entre os pares eles ajudarem mutualmente, entende. Se vejo que isso não funciona, então aí tento proporcionar formação no serviço (REF'1/PORT).

Para os participantes brasileiros, a comunicação é um recurso eficiente para manutenção das interações e cooperação entre os profissionais:

[...] os membros da equipe têm a sua voz, ele consegue [...] a sua área de atuação específica, para a gente poder alocar da melhor maneira recursos que a gente sabe que na área de saúde são poucos [...](REF¹/BR).

[...] Assim, eu tento ouvir, eu tento ser parceira deles, mas quando eu tenho que puxar a orelha eu puxo, eu sou exigente sim [...] (REF²/BR).

[...] do mesmo jeito vem sendo trabalhado com todo o processo de gestão, a gente tem uma regulação é o coração do hospital, e tem uma interligação com todo mundo, a minha ligação, por exemplo, com a chefia de enfermagem enquanto divisão é ímpar, eu preciso da equipe de enfermagem o tempo inteiro, pra admissão, pra entender os processos do paciente quanto à desospitalização, pra no momento da alta, pra transferências, pra exames, enfim, é essa comunicação o tempo inteiro, diante disso, a gente planeja todos os processos[...](REF¹/BR).

\section{Diferentes modelos de gestão e liderança no cuidado}

Destacou-se, no Brasil, a gestão e liderança do enfermeiro como algo que oportuniza o trabalho dos demais profissionais da enfermagem, desde que o enfermeiro tenha a capacidade de liderança e governança na condução dos serviços de saúde. Nessa perspectiva, foi evidenciado um modelo de gestão e liderança participativo, de comunicação horizontalizada, em que se valoriza a motivação da equipe. Das falas emergiu a compreensão de que ambas são fortalecidas ao longo da experiência do enfermeiro: [...]gestão é saber lidar com conflitos, porque é isso que vai, são as habilidades que ela vai ter, técnicas e comportamentais, é que vão fazer com que ela sustente ou não permanecer num espaço como esse [...] (REF $1 / \mathrm{BR})$.

[...]a minha gestão é participativa, ela é aberta, é uma gestão que escuta, de ouvir, de compartilhar, de tomar decisão e encontro [...] (REF²/BR).

[...] em relação aos instrumentos de gestão, à supervisão e à comunicação, a nossa comunicação ela é bem horizontalizada, existe muito diálogo entre a coordenação e a equipe (REF/BR).

As falas dos participantes de Portugal e da Espanha corroboram as afirmativas acima, demonstrando ser necessário implementar uma gestão e liderança do enfermeiro permeadas por compartilhamento e desenvolvimento conjunto:

Eu reconheço a enfermeira diretora até mais como uma líder do que no fundo, como uma gestora, mas acho que tem capacidade de liderança no sentido de envolver todos, procurar envolver todos neste processo, eu acho que cada vez mais há uma tendência de envolvermos na tomada de decisão obviamente dentro do que é possível, mas escutar os profissionais e procurar envolvê-los [...] (REF²/PORT).

[...] a gestão é o primeiro foco necessário nas relações interpessoais, tem que saber qual éo potencial que suas equipes têm e qual são as fragilidades, para que possa extrair o melhor do grupo [...] (REF $\left.{ }^{3} / E S P\right)$.

Em Portugal, o cuidado ao paciente emergiu como um modelo de gestão a ser valorizado, tal como observado na Espanha, em que $o$ atendimento de qualidade e segurança do paciente é reconhecido como primordial. Vale ressaltar que resultado semelhante emergiu, no Brasil, na categoria integração da gestão e liderança no processo de trabalho do enfermeiro, mediante o foco dos enfermeiros nas estratégias frente ao planejamento de treinamentos vislumbrou-se eficiência prestada garantindo a segurança do paciente.

Havia uma confusão, a própria escola não tinha esse modelo que nós seguimos, o que importa aqui não é o modelo, é a abrangência do cuidado que eu presto de forma planeada e consistente para uma abordagem global daquela pessoa que eu tenho ao meu cuidado[...] (REF ${ }^{11} / \mathrm{PORT}$ ).

Bom, o que vem em primeiro lugar é a qualidade do atendimento, a segurança do paciente e a qualidade do atendimento, mas não funcionamos com produtividade $[\ldots]\left(\mathrm{REF}^{1} / \mathrm{ESP}\right)$.

Na Espanha, foi destacada a necessidade de constante atualização do conhecimento por parte da equipe, a fim de 
acompanhar as mudanças nos processos de saúde, em que se assemelha com o resultado da primeira categoria, no Brasil, também com uma ótica dos enfermeiros voltada a uma preocupação na capacitação da equipe.

[...] tem que ser mais versátil e tem que estar atualizado [...] mas as pessoas não têm motivação e não são versáteis [...] então como toda evolução, a mudança é muito importante, e o que Profissionais de saúde como enfermeiros estão atualizados é fundamental na hora de realizar, por exemplo, novas curas, pois novos materiais surgem constantemente e são muitas coisas novas [...] (REF³/ ESP).

\section{DISCUSSÃO}

Ao analisar a categoria precedente, houve a constatação de diferentes percepções nos três países, sendo que em Portugal a comunicação e o diálogo com a equipe foram apontados como facilitadores no processo de gestão da liderança do enfermeiro, em que ele precisa permitir e manter o diálogo horizontalizado com os demais membros da equipe. Na Espanha, a ênfase foi no exercício de liderança nas relações pessoais que incluem o paciente, a família e a equipe de saúde, priorizando a maneira adequada de falar favorece a compreensão do cuidado que será prestado.

No Brasil, identificou-se a preocupação do enfermeiro para o planejamento da assistência e treinamentos que permitam assegurar a qualidade da assistência ao cuidado e motivem a equipe de enfermagem a participar de capacitações e processos educativos como congressos, a fim de favorecer o desenvolvimento do trabalho com eficácia em prol do paciente. Assim, para que esse planejamento ocorra de forma eficiente, é necessária uma comunicação única e alinhada com os membros da equipe.

É possível observar que mesmo com um objeto de trabalho em comum, o cuidado ao paciente, os enfermeiros dos dois países dão seguimento às noções de gestão/liderança de forma diferente, sendo que no Brasil o processo de liderança é pautado em um investimento na equipe, já na Espanha, se atrela ao fluxo de atendimento, trazendo como ponto comum entre eles a comunicação.

Estudo realizado em um hospital no Sul de Minas Gerais trouxe resultados semelhantes, ao mostrar que a comunicação é uma ferramenta essencial do enfermeiro para gerenciamento efetivo de uma organização. A comunicação permite que as atividades ocorram de forma eficiente com o grupo liderado e, nesse sentido, contribui para o desenvolvimento do trabalho e satisfação da equipe ${ }^{16}$.

Nesse sentido, relacionando com a categoria acima, percebe-se a preocupação do enfermeiro em dialogar de maneira horizontalizada com sua equipe, indo ao encontro da burocracia profissional em que valoriza a autonomia dos seus funcionários por meio de seu conhecimento e habilidades e não de maneira autoritária. Isto posto, é oportuno apontar que a burocracia profissional, traz uma pequena necessidade de supervisão direta sobre os profissionais, ela é diferenciada por tornar-se suficientemente autônoma e com baixa hierarquia ${ }^{17}$.

Vale ressaltar o conceito de burocracia profissional de Mintzberg, em que o treinamento requer do profissional longos períodos na universidade ou em instituições especializadas, para adquirir conhecimentos e habilidades específicas de sua profissão. Posteriormente, o local de serviço irá proporcionar novas capacitações, no intuito de aperfeiçoar suas habilidades práticas. Contudo, os treinamentos serão um processo contínuo de novos aprendizados e conhecimento ${ }^{11}$.

Dessa maneira, identificou-se que os enfermeiros brasileiros se preocupam com processos preparatórios contínuos que possam abarcar novos conhecimentos em prol da qualidade da assistência de enfermagem.

As entrevistas com os participantes da Espanha evidenciaram a atenção do enfermeiro ao paciente e seus familiares desde o início de seu tratamento, com amplas explicações abrangendo desde o funcionamento do local da internação até orientações acerca do tratamento. Ficou evidente uma comunicação atenta e realmente preocupada em ouvi-los.

Os líderes de enfermagem são responsáveis por assegurar os melhores cuidados aos pacientes e, para tanto, necessitam trabalhar em estreita colaboração com os pacientes e seus familiares. Assim, cabe ao enfermeiro-líder proporcionar o alcance do objetivo mais importante, ou seja, garantir o bemestar dos pacientes em sofrimento, com tratamento humanizado e particularizado. $\mathrm{O}$ cuidado dos profissionais de enfermagem pode ser notado quando as lideranças de enfermagem facilitam o bom atendimento nas unidades de enfermagem, tal como assinalado em pesquisa internacional ${ }^{18}$. Este resultado constata outro aspecto evidenciado nas entrevistas com participantes da Espanha: os enfermeiros-líderes almejam o bem-estar e a atenção no cuidado dos pacientes e familiares.

Nessa conjuntura, é importante sinalizar que a integração entre a gestão e liderança foi destacada, nesta primeira categoria, pois ambas se complementam, embora se trate de competências diferenciadas. Contudo, um estudo realizado na região Sul do Brasil, indica caminhos na mesma direção deste achado e reforça que para além do cuidar, o trabalho do enfermeiro envolve dimensões diferentes, como administrar/gerenciar, ensino e pesquisa, assim a gestão do cuidado se evidencia como um instrumento de integração do âmbito do trabalho do enfermeiro e associa suas ações considerando a liderança um atributo fundamental para a sua prática ${ }^{19}$.

Também emergiu nos depoimentos a importância do Trabalho em equipe como esteio no processo de gerir/liderar. Nesta, os dados dos três países convergem em relação à compreensão e percepção da confiança entre os profissionais como fator que impulsiona e motiva a equipe.

Nesse sentido, um estudo, corrobora com este achado trazendo que o trabalho em equipe é necessário e constitui um dos componentes estratégicos de enfrentamento da crescente 
complexidade, tanto das necessidades de saúde que requerem uma abordagem ampla e contextualizada como da organização dos serviços e dos sistemas de atenção à saúde em rede ${ }^{20}$.

O relato dos entrevistados da Espanha mostra que a equipe multidisciplinar está envolvida no processo de gestão da equipe, sendo fortalecido de forma mais expressiva o trabalho em equipe que envolve profissionais de diferentes categorias e setores.

Pesquisa afirma que a busca pela integralidade de atenção à saúde e a estruturação de uma equipe multiprofissional transformam este trabalho em unidade produtora de cuidados que precisa de novas formas de mobilização do coletivo que superem o isolamento das práticas dos distintos profissionais e concorram para sua integração. Logo, além de impulsionar e motivar a equipe, o trabalho interprofissional impacta na assistência aos pacientes por ser gerador de um cuidado holístico e com práticas integradas ${ }^{20}$.

Esta percepção foi observada de forma latente nas falas dos entrevistados de Portugal, os quais relataram que a cooperação entre os profissionais gera um apoio mútuo e uma assistência mais qualificada. Identifica-se que, trabalhando em conjunto e de forma articulada, as equipes de saúde ampliam sua capacidade de cuidado e de resolução dos problemas de saúde, uma vez que conseguem tornar os dispositivos de atenção à saúde existentes mais acessíveis, proporcionam uma atenção mais integral e compartilham a responsabilidade pela melhoria da qualidade de saúde e de vida de uma dada população.

No Brasil, percebe-se a adoção de um importante recurso visando mais eficiência: a comunicação. De acordo com alguns autore ${ }^{21}$, o trabalho em equipe em saúde é complexo e considerado impulsionador de transformações, sendo a comunicação efetiva o ponto-chave.

No que se refere aos diferentes modelos de gestão e liderança no cuidado, os resultados demonstram uma relação majoritariamente participativa, englobando líderes, liderados, famílias e outras equipes no desenvolvimento do trabalho, o que diverge da realidade de países com altos índices de subdesenvolvimento, que conservam constructos verticalizados, sem possibilidade de uma tomada de decisão consultiva ou compartilhada. A liderança, nesses casos, ainda é um elemento individualizado, exercido por profissionais médicos de forma estratificada, em que os liderados têm pouca gerência pessoal do trabalho e imperam as relações de poder, coercibilidade e intimidação. Essa realidade pode fragilizar o clima organizacional, sustentar uma ideia de culpa e fomentar uma prestação de serviços mecanicista, na qual relações interpessoais sãodesvalorizadas ${ }^{22}$.

No entanto, os usuários dos serviços de saúde, em geral, se beneficiam mais com um cuidado interdisciplinar, no qual as relações entre os profissionais são amistosas, colaborativas e a comunicação é eficiente, do que com uma assistência fragmentada. Todavia, estudo realizado nos Estados Unidos da América demonstra a persistência de uma relação direcionada da gestão com a equipe médica, desconsiderando o enfermeiro nas decisões, sendo esta uma perda para as equipes de saúde e para os pacientes. O exercício da liderança deve voltar-se ao reforço da importância de todos os profissionais em cada cenário, prestando apoio nas condutas clínicas e fornecendo os meios necessário para a prática profissional. Sabe-se que os enfermeiros, quando usufruem de autonomia, contribuem para o trabalho em equipe, por exercerem influência positiva e aumentarem a eficiência e o engajamento das equipes ${ }^{23}$.

Há experiências exitosas com o fortalecimento da gestão e liderança na enfermagem em cenários críticos com algumas características similares aos países que compõem este estudo, como a imigração, desestrutura no serviço de saúde, déficit de profissionais, índices significativos de pobreza e desigualdade social. O investimento governamental para o desenvolvimento técnico-científico de enfermeiros-líderes e a valorização estrutural e salarial contribuem para a melhoria de todo sistema. Afetase, diretamente, também dimensões como o fortalecimento da educação permanente, mecanismos para comunicação eficiente entre os líderes de diferentes unidades no que se refere a soluções e melhores práticas, planejamento estratégico no cuidado em saúde, melhor distribuição da força de trabalho, transformação de práticas e maior colaboração ${ }^{24}$.

Na perspectiva dos técnicos e auxiliares de enfermagem, a literatura também demonstra que a liderança exercida pelo enfermeiro deve contemplar características como o reconhecimento, motivação, conhecimento, interação e comportamento de líder. Isso reitera as falas dos entrevistados deste estudo, nas quais a liderança democrática foi apontada como um fator essencial por promover um ambiente de trabalho saudável, ser ética e permitir a flexibilização de elementos em prol de melhores resultados, o exercício da criatividade e uma melhor gestão de conflitos.

Nota-se, portanto, que as categorias evidenciadas nesse estudo, trazem características importantes relacionadas com a burocracia profissional de Mintzberg, pois os enfermeiros demonstram o poder do conhecimento, com habilidades gerenciais e de liderança como: a comunicação, escuta, diálogo horizontalizado, planejamento, treinamentos, resolução de problemas, tomada de decisão, participação, entre outras, as quais fortalecem o trabalho em equipe, asseguram a qualidade da assistência ao paciente e familiares, como também permitem ao enfermeiro destacar a sua autonomia no processo de trabalho.

Assim, é válido reforçar que a burocracia profissional é um eixo da estrutura organizacional, a qual envolve a coordenação da força de trabalho em suas tarefas. A mesma se adapta a um ambiente estável, que busca por profissionais com altas habilidades, detentores do controle sobre o seu próprio trabalho e valorizando a autonomia dos profissionais, mediante atividades complexas de uma organização ${ }^{17}$.

\section{CONCLUSÕES E IMPLICAÇÕES PARA A PRÁTICA}

Nos três países, foi possível identificar a importância da comunicação no processo de gerenciamento do enfermeirolíder, pois, por meio dela, ele consegue desenvolver a equipe 
e assegurar a qualidade na assistência prestada ao paciente e familiares.

No Brasil, evidenciou-se uma preocupação com a motivação da equipe, porém no sentido de mantê-la engajada nos treinamentos oferecidos pelas instituições de saúde. Esta postura vai ao encontro da burocracia profissional de Mintzberg, em que o treinamento é contínuo para o aperfeiçoamento profissional, e este ao se capacitar irá fortalecer sua autonomia mediante a prática de suas atividades.

A motivação é fundamental para o trabalho em equipe, por ser um meio de cooperação e aprendizado mútuo, que propicia a visão holística e interdisciplinar do indivíduo e que impacta também na qualidade do cuidado prestado aos pacientes. Tal percepção mostrou-se mais latente na Espanha e em Portugal, enquanto no Brasil a comunicação emergiu como fator determinante para o trabalho em equipe e norteador também da gestão e assistência.

Nos três países analisados, destacaram-se, nos estilos de gestão e liderança, elementos facilitadores, tais como a oportunização da atuação da equipe de enfermagem, habilidades comportamentais para gestão de conflitos e boa comunicação. Foram evidenciados também fatores intervenientes relevantes, como a escuta, clima organizacional, relação interpessoal da equipe, transparência no trabalho e delegação de funções quando apropriado.

Por fim, alerta-se para a necessidade de ampliar os espaços de aprendizado na formação acadêmica sobre a liderança e seus elementos impulsionadores, bem como de reforçar a educação permanente dos profissionais sobre o assunto. É necessário também que as instituições reconheçam e propiciem autonomia para os enfermeiros, com vistas a superar os desafios na gestão em saúde.

No que se refere às limitações do estudo, podemos apontar primeiramente o período pandêmico vivenciado que dificultou em muito o período de coleta de dados, sobretudo, para a realização das entrevistas, nos três cenários do estudo. Em se tratando de um estudo qualitativo, inexiste pretensão ou intenção de generalizações, contudo os achados podem subsidiar os enfermeiros a refletirem sobre suas práticas profissionais na gestão em hospitais universitários.

O fato de somente ser entrevistada uma categoria profissional, pode-se configurar como restrição uma vez que outros profissionais que atuam em hospitais universitários não foram considerados para esta investigação. Outro ponto limitante vincula-se à impossibilidade de generalizações uma vez que foram 3 hospitais universitários em três países, assim qualquer comparação é impossibilitada frente ao universo de hospitais universitários em cada país, podemos ainda indicar que a configuração dos hospitais como de ensino implica características diferenciais em relação aos hospitais que têm outras definições de estrutura organizacional. Nesse sentido, é importante o desenvolvimento de novas pesquisas que abranjam um maior quantitativo de hospitais e enfermeiros.

\section{FINANCIAMENTO}

O presente trabalho foi realizado com apoio do Conselho Nacional de Desenvolvimento Científico e Tecnológico - Brasil (CNPq) - Número: 205736/2018-1.

\section{AGRADECIMENTOS}

Agradecemos aos Hospitais de Ensino das Universidade Federal da Bahia, Castilla-La Mancha e Coimbra por sediaram o estudo e contribuíram com seu desenvolvimento; a Unidade de Investigação em Ciências da Saúde: Enfermagem, da Escola Superior de Enfermagem de Coimbra e enfermeiros, dos serviços de saúde supramencionados pelo tempo disponibilizado e preciosa colaboração.

\section{CONTRIBUIÇÕES DOS AUTORES}

Desenho do estudo. Gilberto Tadeu Reis da Silva. Paulo Joaquim Pina Queirós.

Aquisição de dados. Gilberto Tadeu Reis da Silva.

Análise de dados e interpretação dos resultados. Gilberto Tadeu Reis da Silva. Patrícia Alves Galhardo Varanda. Nívia Vanessa Carneiro dos Santos. Naomy Safira Batista da Silva. Rilary Silva Salles. Simone Coelho Amestoy.

Redação e revisão crítica do manuscrito. Gilberto Tadeu Reis da Silva. Patrícia Alves Galhardo Varanda. Nívia Vanessa Carneiro dos Santos. Naomy Safira Batista da Silva. Rilary Silva Salles. Simone Coelho Amestoy. Giselle Alves da Silva Teixeira. Paulo Joaquim Pina Queirós.

Aprovação da versão final do artigo. Gilberto Tadeu Reis da Silva. Patrícia Alves Galhardo Varanda. Nívia Vanessa Carneiro dos Santos. Naomy Safira Batista da Silva. Rilary Silva Salles. Simone Coelho Amestoy. Giselle Alves da Silva Teixeira. Paulo Joaquim Pina Queirós.

Responsabilidade por todos os aspectos do conteúdo e a integridade do artigo publicado. Gilberto Tadeu Reis da Silva. Patrícia Alves Galhardo Varanda. Nívia Vanessa Carneiro dos Santos. Naomy Safira Batista da Silva. Rilary Silva Salles. Simone Coelho Amestoy. Giselle Alves da Silva Teixeira. Paulo Joaquim Pina Queirós.

\section{EDITOR ASSOCIADO}

Antonio José Almeida Filho (1)

\section{EDITOR CIENTÍFICO}

\author{
Marcelle Miranda da Silva (B)
}

\section{REFERÊNCIAS}

1. Amestoy SC, Backes VMS, Thofehrn MB, Martini JG, Meirelles BHS Trindade LL. Conflict management: challenges experienced by nurseleaders in the hospital environment. Rev Gaúcha Enferm. 2014;35(2):79-85. http://dx.doi.org/10.1590/1983-1447.2014.02.40155. PMid:25158465. 
2. Neves VR, Sanna MC. Concepts and practices of teaching and exercise of leadership in Nursing. Rev Bras Enferm. 2016;69(4):733-40. http:// dx.doi.org/10.1590/0034-7167.2016690417i. PMid:27508480.

3. Oliveira C, Santos LC, Andrade J, Domingos TS, Spiri WC. Leadership in the perspective of Family Health Strategy nurses. Rev Gaúcha Enferm. 2020;41(3):e20190106. http://dx.doi.org/10.1590/19831447.2020.20190106. PMid:32294724.

4. Barreto RMA, Vasconcelos MN, Melo ES, Araújo MAF, Lira RCM, Albuquerque IMAN. Managerial dimensions in the academic education of nurses: an integrative review. Rev Eletr Enf. 2018;20:v20a27. https:// doi.org/10.5216/ree.v20.47945.

5. Leal JAL, Melo CMM. The nurses' work process in different countries: an integrative review. Rev Bras Enferm. 2018;71(2):413-23. http://dx.doi. org/10.1590/0034-7167-2016-0468. PMid:29412301.

6. Nunes EMGT, Gaspar MFM. A liderança em enfermagem e a satisfação dos pacientes em contexto hospitalar. Rev Gaúcha Enferm 2016;37(2):e55726. http://dx.doi.org/10.1590/1983-1447.2016.02.55726. PMid:27253595.

7. Sarik DA, Thompson R, Cordo J, Nieves Roldan I, Gonzalez JL. Good for nurses, good for patients: creating a healthy work environment in a pediatric acute care setting. Nurse Lead. 2020;18(1):30-4. http://dx.doi. org/10.1016/j.mnl.2019.11.005.

8. Fradique MJ, Mendes L. Efeitos da liderança na melhoria da qualidade dos cuidados de enfermagem. Rev Enf Ref. 2013;sérlll(10):45-53. http:// dx.doi.org/10.12707/RIII12133.

9. Thofehrn MB, Montesinos MJL, Jacondino MB, Fernandes HN, Gallo $\mathrm{CMC}$, Figueira $\mathrm{AB}$. Processo de trabalho dos enfermeiros na produção de saúde em um hospital universitário de Murcia/Espanha. Cienc Cuid Saúde. 2015;14(1):924-32. http://dx.doi.org/10.4025/cienccuidsaude. v14i1.22094.

10. Ferracioli GV, Oliveira RR, Souza VS, Teston EF, Varela PLR, Costa MAR Competências gerenciais na perspectiva de enfermeiros do contexto hospitalar. Rev Of Cons Fed Enferm [Internet]. 2020; [citado $2021 \mathrm{ab}$ 9];11(1):15-20. Disponível em: http://revista.cofen.gov.br/index.php/ enfermagem/article/view/2254

11. Mintzberg H. Criando organizações eficazes: estruturas em cinco configurações. São Paulo: Atlas; 1995. 304 p.

12. Taquette SR, Minayo MC. Análise de estudos qualitativos conduzidos por médicos publicados em periódicos científicos brasileiros entre 2004 e 2013. Physis Rev Saúde Coletiva. 2016;26(2):417-34. http:// dx.doi.org/10.1590/S0103-73312016000200005.

13. Cunha MLS, Freire J-M, Repullo JR, Hortale VA. Bureaucratic state and health management training from a historical perspective: similarities and differences between Brazil and Spain. Saude Soc. 2019;28(2):8094. http://dx.doi.org/10.1590/s0104-12902019180616.
14. Major MJ, Magalhães A. Reestruturação do serviço nacional de saúde em Portugal: balanço da empresarialização dos hospitais públicos portugueses. Rev Adm [Internet]. 2014; [citado 2021 abr 9];49(3):476-90. Disponível em: https://www.scielo.br/j/rausp/a/9vc6zNCVxwjYYYJHvmNmmyP /?format=pdf\&lang=pt

15. Costa BRL. Bola de neve virtual: o uso das redes sociais virtuais no processo de coleta de dados de uma pesquisa científica. Rev Interdiscip Gestão Soc [Internet]. 2018; [citado 2021 abr 9];7(1):15-37. Disponível em: http://periódicos.ufba.br/index.php/rigs/article/view/24649/16131

16. Faria CC, Santos MCM, Luz NC, Pereira LF, Lima RS, Haddad JGV. How leader nurse communicates in a hospital: an analysis of discursive practices. Rev Pesqui Cuid é Fundam Online. 2017;9(1):152-8. http:// dx.doi.org/10.9789/2175-5361.2017.v9i1.152-158.

17. Jorge MJ, Carvalho FA, Santiago RP, Ferreira DS. Organização e desempenho avaliação da centralização da patologia do INCA-Brasil. Rev Adm Empres. 2014;54(6):681-91. http://dx.doi.org/10.1590/S0034759020140608.

18. Solbakken R, Bergdahl E, Rudolfsson G, Bondas T. International Nursing caring in nursing leadership: a metaethnography from the nurse leader's perspective. Nurs Adm Q. 2018;42(4):E1-19. http://dx.doi.org/10.1097/ NAQ.0000000000000314. PMid:30180085.

19. Carlos AMM, Kuiava IB, Nogueira MM, Menegon FHA, Andrade SR, Santos JLG. Liderança no ambiente hospitalar: diferenças entre enfermeiros assistenciais e enfermeiros gerentes. Enferm. Foco [Internet]. 2019; [citado 2021 abr 9]; 10(6):143-8. Disponível em: https://pesquisa. bvsalud.org/ portal/resource/pt/biblio-1099617

20. Peduzzi M, Agreli HLF, Silva JAM, Souza HS. Trabalho em Equipe: uma revisita ao conceito e a seus desdobramentos no trabalho interprofissional. Trab Educ Saúde. 2020;18(Supl. 1):e0024678. http:// dx.doi.org/10.1590/1981-7746-sol00246.

21. Pereira RCA, Rivera FJU, Artmann E. O trabalho multiprofissional na Estratégia Saúde da Família: estudo sobre modalidades de equipes. Interface Commun Heal Educ. 2013;17(45):327-40. http://dx.doi. org/10.1590/S1414-32832013005000006.

22. Nogueira JWS, Rodrigues MCS. Effective communication in teamwork in health: a challenge for patient safety. Cogitare Enferm. 2015;20(3):63640. http://dx.doi.org/10.5380/ce.v20i3.40016.

23. Nzinga J, McGivern G, English M. Examining clinical leadership in Kenyan public hospitals through the distributed leadership lens. Health Policy Plan. 2018;33(Supl. 2):ii27-34. http://dx.doi.org/10.1093/heapol/ czx167.

24. Poghosyan L, Liu J. Nurse practitioner autonomy and relationships with leadership affect teamwork in primary care practices: a cross-sectiona survey. J Gen Intern Med. 2016;31(7):771-7. http://dx.doi.org/10.1007/ s11606-016-3652-z. PMid:26951282. 